\title{
Super suppressor
}

We are all familiar with the consequences of loss of tumour-suppressor function, but what about the reverse - is the increased expression of a tumour-suppressor gene a good thing? Manuel Serrano and colleagues have addressed this by generating super transgenic mice that express an additional copy of Ink4a/Arf.

Initially, the authors checked the functionality of the Ink4a/Arftransgene in Ink4a/Arf-null mice and showed that the transgene is regulated like the endogenous gene both during development and in response to oncogenic stress. So, Serrano and colleagues investigated the function of super Ink4a/Arf - where one copy of the transgene is present in addition to the endogenous Ink4a/Arf wildtype loci - in mouse embryo fibroblasts (MEFs) and mice.

The immortalization of cells in culture is commonly associated with loss of either $\mathrm{p} 53$ or ARF function, aiding the cells to bypass senescence. After twenty-five rounds of cell passage, 95\% of the wild-type MEFs, compared with only $40 \%$ of super Ink4a/ArfMEFs, were immortalized. In becoming immortalized, wild-type MEFs showed either loss of p53 or ARF function, whereas the transgenic MEFs lost p53 function only, indicating that the extra copy of Arf was protective. Moreover, super Ink4a/Arf MEFs were more resistant to transformation by either the adenovirus oncogene E1A or oncogenic HRAS-V12.
Are similar results evident in the super Ink4a/Arf mice? Treatment of these animals with a range of carcinogens resulted in reduced tumour formation compared with wild-type littermate controls. Importantly, like Serrano's super p53 mice, the expression of the additional copy of Ink $4 a /$ Arf did not affect the ageing process - other transgenic mice overexpressing deregulated p53 have aged prematurely.

Serrano and colleagues conclude that the increased expression of tumour-suppressor genes is protective against tumour formation and that this overexpression is unlikely to be detrimental as long as the gene is regulated and expressed in a manner similar to the endogenous gene. Recent publications have indicated human allelic variability in mRNA expression levels, and variable duplicated DNA sequences within the genome, implying that there might be individuals with increased expression of tumour-suppressor genes and a genetically inherited resistance to cancer.

Nicola McCarthy

\section{(2) References and links} ORIGINAL RESEARCH PAPER Matheu, A. et al. Increased gene dosage of Ink4a/Arf results in cancer resistance and normal aging. Genes Dev. 1 Nov 2004 (doi:10.1101/gad.310304)

FURTHER READING Campisi, J. Cancer and ageing: rival demons. Nature Rev. Cancer $\mathbf{3}$, 339-349 (2003) | Garcia-Cao, I. 'Super p53' mice exhibit enhanced DNA damage response, are tumour resistant and age normally. EMBO J. 21, 6225-6235 (2002)

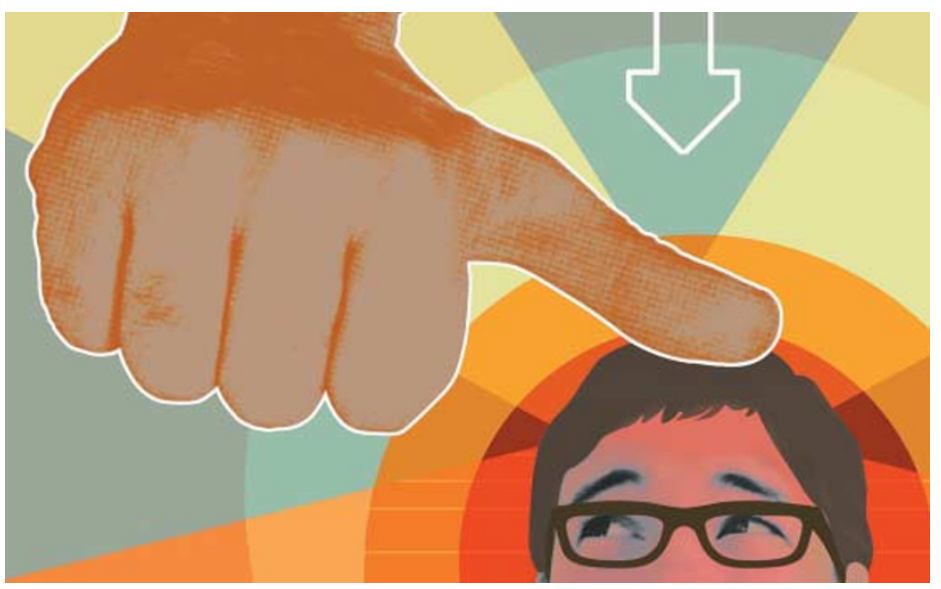

\section{PROTEIN KINASES}

Tumour cell and endothelial cell therapy of oral cancer by dual tyrosine kinase blockade.

Yigitbasi, O. G. et al. Cancer Res. 64, 7977-7984 (2004)

Activation of the epidermal growth factor (EGF) signalling pathway is associated with the progressive growth of head and neck cancer. The tyrosine-kinase inhibitor AEE788 blocks EGF and vascular endothelial growth factor signalling pathways, so the authors investigated its efficacy in inhibiting the growth of two human head and neck cancer cell lines injected into nude mice. AEE788 significantly inhibited tumour-cell growth and induced apoptosis in both endothelial and tumour cells. So, patients with head and neck cancer should be included in clinical trials of AEE788.

\section{IMMUNOLOGY}

SOCS1 is a key brake of antigen presentation by dendritic cells and restricts anti-tumour immunity.

Shen, L. et al. Nature Biotechnol. 21 Nov 2004 (doi:10.1038/nbt1035)

The successes so far for tumour vaccines have been limited, for many and various reasons. In this paper the authors show that the suppressor of cytokine signalling 1 (SOCS1) is a key regulator of antigen presentation by dendritic cells. Silencing SOCS1 expression strongly enhances antigen-specific antitumour immunity.

\section{THERAPEUTICS}

\section{A polymer library approach to suicide gene therapy} for cancer.

Anderson, D. G. et al. Proc. Natl Acad. Sci. USA 101, 16028-16033 (2004)

Effective suicide-gene therapy requires that a toxin is delivered to tumours with little effect on normal cells. One huge hurdle is finding a vector that is itself non-toxic; Sawacki and co-workers are investigating biodegradable poly ( $\beta$-amino esters) as alternatives to viral vectors or non-viral systems. They screened a library of more than 500 biodegradable poly ( $\beta$-amino esters) and identified one - C32 - that is non-toxic and avoids gene expression in healthy muscle, and is highly efficient at delivering DNA to tumour cells.

\section{ANGIOGENESIS}

Requirement for sphingosine 1-phosphate-1 in tumor angiogenesis demonstrated by in vivo RNA interference.

Chae, S.-S. et al. J. Clin. Invest. 114, 1082-1089 (2004)

Angiogenesis requires the lipid mediator sphingosine 1phosphate (S1P) and its receptor S1P1. Chae et al. show that S1P1 is strongly induced in tumour vessels, and, using small interfering RNA (siRNA) for S1P1, that downregulation of S1P1 suppresses vascular stabilization, angiogenesis and tumour growth in vivo. The authors suggest that S1P1 siRNA might provide a novel therapeutic approach for the control of pathogenic angiogenesis. 